Erkenntnisse aus einer bundesweiten Befragung

\section{Kommunale Wirtschaftsförderung und Nachhaltigkeit}

\author{
Nachhaltigkeit ist als Thema in der kommunalen Wirtschafts- \\ förderung durchaus von Interesse. Allerdings zeigen die \\ Ergebnisse einer deutschlandweiten Befragung, dass bestehende \\ Gestaltungsspielräume noch nicht erkannt und noch nicht \\ ausreichend genutzt werden. \\ Von Jürgen Bunde, Josef Rother und Stefan Blümling
}

W enn es um Nachhaltigkeit auf kommunaler Ebene geht, geraten in erster Linie die Maßnahmen der Stadt und des Landkreises in den Blick. Auch die privaten Haushalte, die kommunalen Eigenbetriebe und die Bildungsträger werden einbezogen. Deutlich weniger werden die lokalen Unternehmen adressiert. Dabei gibt es in nahezu jeder deutschen Kommune ab 20.000 Einwohner eine Wirtschaftsförderung, entweder als verwaltungsinterne Stelle oder als externe Gesellschaft mit öffentlichem Auftrag organisiert.

\section{Bundesweite Befragung}

Traditionelle Aufgabe der Wirtschaftsförderung ist es, mit einem umfangreichen Instrumentarium die Standortökonomie zu stärken, neue Unternehmen anzuziehen und die bestehenden kommunalen Gewerbeimmobilien bestmöglich in Nutzung zu bringen. Die Wirtschaftsförderung steht deshalb häufig in gutem Kontakt $\mathrm{zu}$ den ansässigen Betrieben und ist erste Anlaufstelle für ansiedlungswillige Unternehmen. Mit dieser Aufgabenbeschreibung sind die kommunalen Wirtschaftsförderer als der wichtigste Impulsgeber für eine nachhaltigere Orientierung der Unternehmen prädestiniert. Aber wie ist es um die Wahrnehmung dieser Rolle bestellt?

Um dieser Frage nachzugehen, hat die GEFAK, Gesellschaft für angewandte Kommunalforschung $\mathrm{mbH}$, in Kooperation mit verAntworten 2014 eine bundesweite Online-Befragung bei rund
1.000 Wirtschaftsförderungseinrichtungen durchgeführt. Es wurde untersucht, welche erfolgreichen Projekte zu diesem Thema bereits realisiert wurden, welche Hemmnisse bestehen und wie die Resonanz bei den ansässigen Unternehmen war. Mit einem Rücklauf von 29 Prozent liegen zu diesen Fragen nun profunde Erkenntnisse vor. Die Antworten ergeben ein gemischtes Bild.

\section{Nachhaltige Unternehmen als Nischenthema}

Eine Unterstützung nachhaltig aufgestellter Unternehmen im Rahmen der Bestandspflege oder der Ansiedlungspolitik ist nur für etwa für ein Fünftel der Wirtschaftsförderer ein Thema. Ansonsten dominieren klassische Aspekte wie die Vermarktungsmöglichkeit der eigenen Gewerbeflächen sowie die Aussicht auf Gewerbesteuereinnahmen und auf neue Arbeitsplätze am Standort.

Die bevorzugte Nutzung von Gewerbebestandsflächen gegenüber Flächen auf der sogenannten grünen Wiese ist ein kontroverses Thema. Zwar geben knapp 60 Prozent der Befragten an, es gebe eine solche Priorisierung, in der Umsetzung jedoch gelinge dies in drei von vier Fällen nur teilweise oder gar nicht. Dieses Umsetzungsdefizit begründen die Befragten damit, dass die Bestandsflächen nicht zu den Anforderungen der Investoren passen, die Kosten der Bestandsflächen-Reaktivierung zu hoch seien und die interkommunale Konkurrenz diese Priorisierung konterkariere.
Der wichtigste Themenstrang, dem sowohl vonseiten der Wirtschaftsförderung als auch der Unternehmen besondere Bedeutung beigemessen wird, ist die Fachkräftesicherung. Die durch die Wirtschaftsförderer dort verfolgten nachhaltigen Lösungsansätze richten sich auf die Kooperationsförderung zwischen Hochschulen beziehungsweise Schulen und Wirtschaft. Weitere Ansätze fokussieren auf besondere Qualifizierungsmaßnahmen für Jugendliche, Frauen, Migranten, Menschen mit Behinderung und ältere Mitarbeiter. Ebenso unterstützt die Wirtschaftsförderung häufig Unternehmen bei der mitarbeiterfreundlichen Ausgestaltung der Arbeitsverhältnisse.

\section{Nachhaltigkeit als strategisches Ziel}

Insgesamt gesehen gibt es noch viel Handlungspotenzial für die Wirtschaftsförderung, dem Thema Nachhaltigkeit eine größere strategische Bedeutung beizumessen. Doch dafür muss der politische Wille vorhanden sein. Ob Nachhaltigkeit als strategisches Ziel der kommunalen Wirtschaftsentwicklung gesehen wird, kann zumindest bezweifelt werden, denn eine klare Nachhaltigkeitsstrategie wird nur in einem Drittel der Kommunen verfolgt. Und von diesen hat wiederum nur ein knappes Drittel bestätigt, dass die Strategieumsetzung mit Kennziffern gesteuert wird.

AUTOREN + KONTAKT

Dr.Jürgen Bunde ist Geschäftsführer und Josef Rother ist Prokurist der Gesellschaft für angewandte Kommunalforschung mbH (GEFAK).

GEFAK, Gesellschaft für angewandte Kommunalforschung $\mathrm{mbH}$, Ockershäuser Allee 40b 35037 Marburg. Tel.: +49 6421 1728-0, E-Mail: info@gefak.de, Internet: www.gefak.de.

Dr. Stefan Blümling ist freiberuflicher Berater,

Coach und Dozent für Corporate Social Responsibility und Transformationsthemen.

verAntworten - Forschung und Beratung für Nachhaltigkeit, Heinrich-Heine-Straße 11 a 35039 Marburg. Tel.: +49 1752327855 , E-Mail: bluemling@verantworten.com, Internet: www.verantworten.com 